\title{
The use of fan scrapers: Microwear evidence from Late Pottery Neolithic and Early Bronze Age, Ein Zippori, Israel
}

\author{
Richard W. Yerkes ${ }^{1}$, Ran Barkai ${ }^{2}$, Avi Gopher ${ }^{2}$, Katia Zutovski ${ }^{2}$ \\ 1. Department of Anthropology, Ohio State University. 4034 Smith Lab., 174 W. 18th Ave., Columbus, OH \\ 43210-1106, USA. Email: yerkes.1@ osu.edu \\ 2. Tel Aviv University, Dept. Archaeology and Ancient Near Eastern Cultures, and Sonia and Marco Nadler \\ Institute of Archaeology, Tel-Aviv University, Tel-Aviv 69978, Israel. Email: Barkai: barkaran@ post.tau.ac.il; \\ Gopher: agopher@post.tau.ac.il; Zutovski: katiarina81@gmail.com
}

\begin{abstract}
:
The results of a microwear analysis of samples of fan scrapers and fan scrapers spalls from late Pottery Neolithic (PN) and Early Bronze Age (EBA) occupation layers at Ein Zippori, Lower Galilee, Israel are presented. The goal of the microwear analysis was to determine the function of the fan scrapers and compare the visible usewear on the scrapers found in late PN and EBA lithic assemblages. The results indicate that during both periods most of the fan scrapers were used to skin and butcher animals, while some were also used for hide processing and bone working. The working edges of the fan scrapers had sharp, moderate, or steep edge-angles, and different edges were used for different tasks. Edges with microwear from scraping meat, bone, and hides (including some hides that may have been treated with abrasives) had steep edge-angles, while there were moderate or sharp edge-angles on the edges of fan scrapers used for cutting. Two sub-types of fan scrapers were identified, flat cortex fan scrapers (FCFS), and cortical fan scrapers (CFS) with convex dorsal faces. The CFS were abundant in PN contexts, while the FCFS were more common in EBA layers. However both of the sub-types had similar microwear traces.
\end{abstract}

Keywords: microwear analysis; late Pottery Neolithic; Wadi Rabbah; Early Bronze Age; southern Levant

\section{Introduction}

This microwear analysis of 31 fan scrapers and 14 fan scraper spalls from the late prehistoric site of Ein Zippori, Israel complements the techno-typological study of 191 fan scrapers and 35 fan scraper spalls from the site (Zutovski et al. 2016). The microwear sample includes $16 \%$ of the fan scrapers and $40 \%$ of the fan scraper spalls in the techno-typological assemblage.

Published by the School of History, Classics and Archaeology, University of Edinburgh ISSN: 2055-0472. URL: http://journals.ed.ac.uk/lithicstudies/

This work is licensed under a Creative Commons Attribution 2.5 UK: Scotland License. 
Ein Zippori was excavated by the Israel Antiquities Authority between 2011 and 2013. Eleven large blocks covering ca. $5000 \mathrm{~m}^{2}$ (Areas A - N,) exposed a stratified sequence of mainly late Pottery Neolithic (PN) Wadi Rabah (WR), and Early Bronze Age (EBA) 1b layers. In some areas, Pre-Pottery Neolithic (PPN) and post WR-pre Chalcolithic Ghassulian (CHG) layers were exposed (Milevski et al., 2014).

Microwear results help us understand the role of fan scrapers in late PN and EBA socioeconomic systems. This study is another example of how changes in stone tool technology and function can be used as an index for cultural development during those periods (Barkai \& Gopher 2012; Levy 2003; Marder et al. 1995; Rosen 1997; Rosen \& Vardi 2014; Yamada 2012; Yerkes \& Barkai 2012).

\subsection{Fan scrapers}

Fan scrapers are unifacial tools made on broad thin cortical flakes. They have also been called "tabular fan scrapers" and "Ghassulian fan scrapers," and are diagnostic CHG and EBA tools in the southern Levant (Bankirer and Marder 2003; Barkai 2004: 90; Barkai and Gopher 2012: 837; Bar-Yosef et al. 1977; Beit-Arieh 2003; Crowfoot-Payne 1983: 720-722; Fujii 1999; Greenhut 1989; Levy and Alon 1985; Mallon 1932; Mallon et al. 1934; McConaughy 1979: 69, 301-304; 1980: 53-54; Marder et al 1995; Noy 1998; Rosen 1983; 1997: 78-79, 2013: 402; Rosen and Vardi 2014: 333; Rowan and Levy 1991: 117; Stepieǹ 2012; UngerHamilton 1991: 149; van den Brink et al 2004). They are also present in late PN lithic assemblages (Barkai and Gopher 2012: 837; Gopher 1989: 119; Kaplan 1969; Khalaily 2011; Khalaily and Nagorsky 2013; Yevin and Olami 1979).

Some fan scrapers from CHG and EB sites in the southern Levant were made at workshops near sources of fine-grained flint in Sinai, Negev, and Jordan (Fujii 1999; MüllerNeuhof 2006; Quintero et al. 2002; Rosen 1983; 1997: 71-80; Rowan and Levy 1991). More fan scrapers are found at pastoral nomad sites near lithic sources than at settled villages (Beit Arieh 2003; Fujii 1999; Rosen 1983). Fan scrapers are associated with livestock management and may be related to secondary products of domestication (Fijii 1999; Halstead and Isaakidou 2011; Quintero et al. 2002; Sherratt 1997a; 1997b).

\subsubsection{Previous research on the functions of fan scrapers}

Proposed functions for fan scrapers include wood-working, plant processing, shearing sheep, and butchering animals (Baird 2001: 649; Barket and Bell 2011; Bennett et al. 1989; Elliot 1977; Henry 1995: 372-373; Rosen 1997: 74; Rosen and Vardi 2014: 333; Stepieǹ 2012; Unger-Hamilton 1991: 152). Quintero et al. (2002: 46) suggest that they were handheld "cortical flake knives" designed to be re-sharpened easily and used for many activities. Fan scrapers did not replace "typical" side and end scrapers. Were they meant to be used for special tasks? A study of five side and end scrapers selected randomly from Ein Zippori was conducted to see if typical scrapers were used for different activities than fan scrapers. They were not. All five scrapers were also used to scrape and cut hide, meat, and bone - just like fan scrapers (see below). However, symbolic or ceremonial functions have been suggested, based results of low-power usewear studies and recovery of fan scrapers from ritual contexts (McConaughy 1979; 1980; Zutovski et al. 2016).

There have been few microwear studies. McConaughy's (1979; 1980) low-power microwear analysis of artifacts from Early Bronze Age Bâb edh-Dhrâ, Jordan included 12 fan scrapers and fragments. He compared usewear on fan scrapers with wear traces from his replicative experiments (1979: 39) and descriptions of microwear (Semenov 1964: 85-93). He found no edge-rounding or "edge-polishing" from scraping hide or wood on the Bâb edhDhrâ fan scrapers, but did see evidence for bone or hard wood cutting. Polish back from the 
edges of two scrapers suggested use on soft materials like "flesh." Striations were oriented obliquely to working edges. He concluded that fan scrapers were mainly used for butchering (1979: 69, 301-304). Later McConaughy (1980: 53-54) suggested that fan scrapers may have been used in ritual ceremonies in sanctuaries. Others cited McConaughy's interpretations to support claims that fan scrapers were ritual knives (Elliott 1977; Rosen 1997: 74; Rosen and Vardi 2014: 333; but see Stepién 2012).

Rowan and Levy (1991) examined 10 Chalcolithic fan scrapers from Shiqmim, Israel in a study of 197 scrapers. They said that both high-power and low-power magnification was used in their study (1991: 121-122), but they did not use incident light high-power metallurgical microscopes. They recorded edge-damage, rounding, and striations, but were not able to identify microwear polishes. They classified three fan scrapers and fragments as cutting tools, four as scrapers, and one as a knife-scraper. They concluded that these fan scrapers were used to cut or scrape hide or meat. Distal edges on two fan scrapers were missing (Rowan and Levy 1991: 131-132).

Unger-Hamilton's (1991) high-power examination of a single triangular or oval fan scraper with an "accidental" notch, but no bulb of percussion, from Jawa, Jordan was limited since the context of the fan scraper was not clear, and there were no photomicrographs. Unger-Hamilton noted that no edge-damage, and striations perpendicular or oblique to distal edges revealed it had been used to scrape material that produced a "buoyant looking" polish. The best match that Unger-Hamilton found in her comparative collection were experimental scrapers used on reeds (1991: 152). However, there are no striations on experimental reed scrapers. Wear traces on the Jawa fan scraper could be from plants. However, van Gijn (1990: 27,30 , fig. 13b) found that when powered ochre and a tanning agent were added to hides with thick subcutaneous fat layers, bright polish with striations forms. This polish resembles microwear from cutting reeds (van Gijn 2010: fig. 4.6d), but there are striations on "ochre hide" scrapers, and wear forms along every protrusion and indentation. When reeds are cut or scraped polish only forms on protrusions (van Gijn 1990: 30; 2010: 66).

Barket and Bell (2011) sheared Hampshire and Suffolk sheep with fan scraper replicas to see if they had been used for that task (Bennett et al. 1989; Henry 1995). A low-power image (20x) shows microwear similar to traces from scraping fresh hide or meat or plants. Highpower examination may reveal distinctive sheep-shearing microwear, but sheep with thick fleece like Hampshire and Suffolk breeds may not have been around during the late PN or EBA (Davis 1987: 156-162; Halstead and Isaakidou 2011: 63-64, 67-68).

McConaughy's observations were very astute. His study was before high-power microwear techniques had been refined. Nonetheless, he noted that while fan scrapers looked like heavy-duty scrapers, most were used as hand-held butchering knives (1979: 301-304). Rowan and Levy (1991) agreed with his assessment. There is less support for use of fan scrapers to scrape reeds or shear sheep.

\section{Materials and methods}

A sample of 31 fan scrapers and fragments and 14 fan scraper spalls from Ein Zippori were selected for microwear analysis. They came from seven of 11 Excavation Areas at Ein Zippori (B, C, D, E, F, L, and N). There is no difference in frequencies of fan scrapers and spalls in the microwear sample and frequencies of all recovered fan scrapers and spalls from these areas $($ Chi-square $=3.77 ; \mathrm{T}(0.001)=22.46$, 6df). Several fan scraper sub-types have been described (Rosen, 1983, 1997: 71-80; Rowan and Levy 1991), but the classification by Zutovski et al. (2016) for the larger sample of 191 fan scrapers is used here. This fan scraper typology includes a "broad and continuous array of sizes and morphological configurations" of scrapers that have been classified as tabular scrapers (Quintero et al. 2002: 17). Most, but 
not all, fan scrapers were made on flint nodules with cortical surfaces (Rosen 1983). They may be oval, elongated, fan shaped, round, or irregular in shape, with abrupt to sharp edgeangles (Zutovski et al. 2016). There was no significant difference in the frequencies of different shaped fan scrapers in the microwear sample and in the larger assemblage (Chisquare $=9.13 ; \mathrm{T}(0.001)=20.52,5 \mathrm{df})$.

While fan scrapers from late PN layers of Ein Zippori are from good Wadi Rabbah contexts, some from EBA layers were mixed with artifacts from the underlying late PN layers, probably the result of building activities. A combined 'flint-pottery-stratigraphy' approach was used to separate "clean" contexts from mixed layers (Tables 1 and 2). Only 22 fan scrapers and nine fan scraper spalls with identifiable microwear are shown on Tables 1 and 2. Two other fan scrapers had generic weak traces (the type of material worked could not be determined). Seven fan scrapers and five spalls had no visible microwear.

In the microwear sample (31 fan scrapers, 14 spalls), 19 scrapers and five spalls were from Late PN contexts, 10 scrapers and nine spalls were from EBA layers, and two scrapers with no visible microwear (not shown on Tables 1 and 2) were from mixed contexts. Not counting those two, $56 \%$ are from late PN layers and $44 \%$ are from EBA layers. There is no significant difference between frequencies of scrapers from each context in the microwear sample and in the techno-typological assemblage of 118 fan scrapers and spalls (Chi-square = 9.2; $\mathrm{T}(0.001)=10.83)$. In the techno-typological study, $89.5 \%$ of cortical fan scrapers (CFS), which had convex cortical dorsal faces, were from late PN contexts, while $61 \%$ of flat cortex fan scrapers (FCFS), which have flat dorsal faces covered with cortex, were in EBA layers (Zutovski et al. 2016). In the microwear sample of 31 fan scrapers, $74 \%$ of the CFS scrapers were from late PN layers and 50\% of the FCFS scrapers were from EBA contexts. There was no significant difference between the frequencies in the microwear sample and in the larger Fsquare $=6.22 ; \mathrm{T}(0.001)=16.27,2 \mathrm{df})$. It should be noted that when flatness of the dorsal face was indeterminate, they were classified as CFS (Zutovski et al. 2016).

\subsection{Methods of microwear analysis}

Fan scrapers and spalls from Ein Zippori were examined to determine tool motions, and: (1) if they were utilized or hafted, (2) which edges were used, and (3) what materials were worked. "High-power" incident light methods developed by Semenov (1964) and Keeley (1980) were employed. Both "low-power" and "high-power" techniques provide information, but low-power methods do not allow discrimination between specific types of worked materials (Juel Jensen 1988; Longo and Skakun 2008; Yerkes and Kardulias 1993). Fan scrapers and spalls were examined at Tel Aviv University with a Nikon Optiphot metallurgical microscope at magnifications of 50x, 100x, and 200x, and at Ohio State University at magnifications of $62 \mathrm{x}, 187.5 \mathrm{x}$, and 375x using an Olympus BHM microscope with a digital camera. Prior to cleaning, scrapers were examined for residues (none were visible). They were immersed in liquid detergent in a sonic cleaner to remove dirt and postdepositional handling traces (finger grease). Some were immersed in a $10 \%$ solution of HCL in the sonic cleaner to try and remove caliche (this was not successful). After cleaning, acetone was used to remove any finger grease from mounting on the microscope stage. Microwear on Ein Zippori artifacts were compared with patterns on flint tools in a reference collection of experimental flint tools (including heat-treated replicas) that were used for a variety of tasks, and on published photomicrographs of microwear traces (Keeley 1980; van Gijn 1990, 2010). The results (Figure 1) were used to test hypotheses about fan scraper functions and their role in late prehistoric economic systems. 


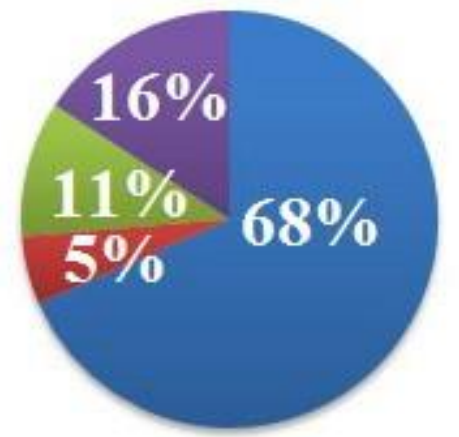

late Pottery Neolithic (Wadi Rabah) $\mathbf{n}=19$

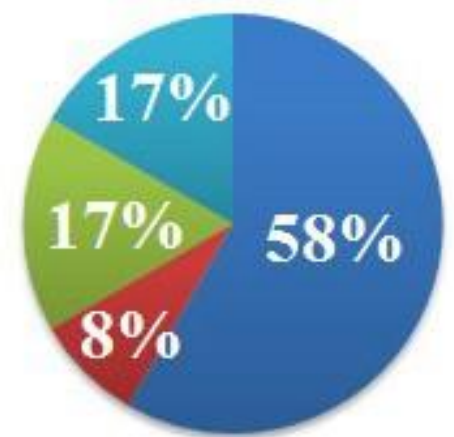

Early Bronze Age

$\mathrm{n}=\mathbf{1 2}$

Tool Functions:

$\square$ Cut and scrape meat, fresh hide and bone

$\square$ Cut, scrape, and saw meat, fresh hide and bone

$\square$ Cut, scrape hide or meat

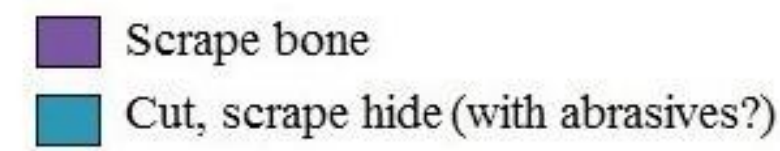

Two fan scrapers from late Pottery Neolithic levels with weak generic microwear are not included.

Figure 1. Microwear on utilized late pottery Neolithic and Early Bronze Age fan scrapers and spalls from the Ein Zippori Site, Isreal. Frequencies of different tool functions for utilized fan scrapers and fan scraper spalls in late Pottery Neolithic (PN) and Early Bronze Age (EBA) layers Ein Zippori. Chi-square test showed no significant difference between periods. Most fan scrapers were used for skinning and butchering animals and processing hides. Scrapers from late PN levels were also used to scrape bone, and scrapers from EBA levels were also used to scrape hides with thicker fat layers that may have been treated with abrasives. From Tables 1 and 2 . Two fan scrapers from late PN levels with weak generic microwear are not included.

\section{Results of the microwear analysis}

Nearly all of the fan scrapers $(90 \%)$ and spalls $(86 \%)$ had cortex on their dorsal face. Many also had patches of caliche on their faces, or were patinated. It was possible to identify and interpret microwear on 22 of 31 examined fan scrapers (71\%), and on 9 of 14 fan scraper spalls $(64 \%)$. Two other fan scrapers had generic weak microwear traces on their edges, and the type of worked material could not be determined. The results are summarized in Figure 1 and Tables 1 and 2. Examples of microwear on utilized fan scrapers and spalls are shown in Figures 2 to 8 . Utilized fan scrapers and spalls were usually used to work more than one type of material. Most were used for skinning and butchering animals and processing hides. Scrapers from late PN levels were also used to scrape bone, and scrapers from EBA levels were also used to scrape hides with thicker fat layers that may have been treated with abrasives, but there was no significant difference in fan scraper function during both periods (Chi-square $=5.73 ; \mathrm{T}(0.001)=118.47$, 4df, see Figure 1$)$.

There were no obvious hafting traces on any utilized fan scrapers. Two fan scrapers had prehension traces (MW\#5 and 6) (Tables 1 and 2), but all utilized fan scrapers probably were held in the hand. Most utilized scrapers had wear on several edges. Eight (36\%) had it on three edges, seven $(32 \%)$ had wear on two edges, four (18\%) had wear on all four edges, and three $(14 \%)$ had wear on one edge. Retouched and unretouched edges were used, and notches on three scrapers (MW\#10, 15, 21) were used to scrape meat off of bone or to make bone tools (Tables 1, Figure $2 \mathrm{~A}$ and B). 
Table 1. Ein Zippori fan scrapers microwear results. Abbreviations: Loc. = Locus, Buck. = Bucket; late PN = late Pottery Neolithic Wadi Rabbah, EBA = Early Bronze Age1b, cultural period based on stratigraphy and pottery analysis, TS = mixed surface; FCFS = flat cortex fan scraper with flat dorsal face, CFS $=$ cortical fan scraper with convex dorsal face; $\mathrm{GL}=$ greatest length, $\mathrm{GW}=$ greatest width, $\mathrm{Th}=$ thickness in $\mathrm{mm}$; elong. = elongated, frag. $=$ fragment, irreg. $=$ irregular shapes; $\mathrm{m}=$ moderate, srp = sharp, stp = steep edge-angles.

\begin{tabular}{|c|c|c|c|c|c|c|c|c|c|c|c|c|c|c|c|}
\hline $\begin{array}{l}\text { MW } \\
\#\end{array}$ & Locus & Bucket & $\#$ & Area & $\begin{array}{l}\text { Cultural } \\
\text { Context }\end{array}$ & $\begin{array}{l}\text { Layer } \\
\text { according to } \\
\text { stratigraphy }\end{array}$ & $\begin{array}{c}\text { Period } \\
\text { according to } \\
\text { pottery } \\
\text { analysis }\end{array}$ & $\begin{array}{l}\text { FCFS } \\
\text { - CFS }\end{array}$ & Length & Width & Thick & $\begin{array}{l}\text { Scraper } \\
\text { Shape }\end{array}$ & EA & Motions & $\begin{array}{l}\text { Worked } \\
\text { Material }\end{array}$ \\
\hline 1 & 1321 & 13185 & 3 & $B$ & EBA1b & EBA & EBA & CFS & 5 & 6.5 & 1.4 & fan & sharp, $m$ & $\begin{array}{c}\text { cut* } \\
\text { scrape }\end{array}$ & $\begin{array}{l}\text { meat, fresh } \\
\text { hide, bone }\end{array}$ \\
\hline 2 & 1417 & 13450 & & B & EBA1b & TS & EBA & CFS & 6.1 & 6.8 & 1.3 & fan & moderate & $\begin{array}{l}\text { cut? } \\
\text { scrape }\end{array}$ & $\begin{array}{l}\text { meat, fresh } \\
\text { hide, bone }\end{array}$ \\
\hline 3 & 1427 & 13471 & 2 & B & EBA1b & EBA & Mixed & CFS & 5.1 & 2.2 & 1 & elongated & srp, m & $\begin{array}{c}\text { cut, } \\
\text { scrape, } \\
\text { saw }\end{array}$ & $\begin{array}{l}\text { meat, fresh } \\
\text { hide, bone }\end{array}$ \\
\hline 4 & 3537 & 14954 & & B & late PN & late PN & late PN & FCFS & 8.5 & 7.6 & 1.6 & oval & moderate & $\begin{array}{c}\text { cut? } \\
\text { scrape }\end{array}$ & $\begin{array}{l}\text { meat, fresh } \\
\text { hide, bone }\end{array}$ \\
\hline 5 & 3538 & 15055 & & B & late PN & late PN & late PN & CFS & 6.6 & 5.8 & 1.8 & oval & $\begin{array}{l}\text { sharp, } \\
\text { steep }\end{array}$ & scrape & bone \\
\hline 6 & 3561 & 15133 & & B & late PN & late PN & late PN & CFS & 6.5 & 5.9 & 2.2 & fragment & moderate & $\begin{array}{c}\text { cut, } \\
\text { scrape, } \\
\text { saw }\end{array}$ & $\begin{array}{l}\text { meat, fresh } \\
\text { hide, bone }\end{array}$ \\
\hline 8 & 1941 & 17618 & & C & late PN & late PN & late PN & FCFS & 6.4 & 8.8 & 2 & fan & sharp, m & $\begin{array}{c}\text { cut, } \\
\text { scrape }\end{array}$ & $\begin{array}{l}\text { meat, fresh } \\
\text { hide, bone }\end{array}$ \\
\hline 9 & 1944 & 17825 & & C & late PN & late PN & late PN & CFS & 7.5 & 6 & 1.8 & $\begin{array}{c}\text { oval } \\
\text { (fragment) }\end{array}$ & m, steep & $\begin{array}{l}\text { cut, } \\
\text { scrape }\end{array}$ & $\begin{array}{l}\text { meat, fresh } \\
\text { hide, bone }\end{array}$ \\
\hline 10 & 1976 & 17805 & & C & late PN & late PN & late PN & CFS & 4.1 & 6.3 & 1.4 & $\begin{array}{l}\text { frag. with } \\
\text { notch }\end{array}$ & steep & scrape & bone (meat) \\
\hline 11 & 1996 & 90051 & & C & late PN & late PN & late PN & FCFS & 4.5 & 4.1 & 1.4 & round & moderate & scrape & bone \\
\hline 12 & 1996 & 90039 & & C & late PN & late PN & late PN & CFS & 5.1 & 3.2 & 1 & oval & sharp & $\begin{array}{l}\text { cut, } \\
\text { scrape }\end{array}$ & $\begin{array}{l}\text { meat,fresh } \\
\text { hide,bone? }\end{array}$ \\
\hline 13 & 9013 & 90058 & 2 & $\mathrm{C}$ & late PN & late PN & late PN & CFS & 5.1 & 4.5 & 1.1 & oval & sharp & $\begin{array}{l}\text { cut, } \\
\text { scrape }\end{array}$ & $\begin{array}{l}\text { meat, fresh } \\
\text { hide, bone }\end{array}$ \\
\hline 14 & 9013 & 90080 & & C & late PN & late PN & late PN & CFS & 5.4 & 4.7 & 1 & $\begin{array}{c}\text { oval } \\
\text { (fragment) }\end{array}$ & m, steep & $\begin{array}{l}\text { cut, } \\
\text { scrape }\end{array}$ & $\begin{array}{l}\text { meat, fresh } \\
\text { hide, bone }\end{array}$ \\
\hline
\end{tabular}




\begin{tabular}{|c|c|c|c|c|c|c|c|c|c|c|c|c|c|c|c|}
\hline $\begin{array}{l}\text { MW } \\
\#\end{array}$ & Locus & Bucket & $\#$ & Area & $\begin{array}{l}\text { Cultural } \\
\text { Context }\end{array}$ & $\begin{array}{l}\text { Layer } \\
\text { according to } \\
\text { stratigraphy }\end{array}$ & $\begin{array}{l}\text { Period } \\
\text { according to } \\
\text { pottery } \\
\text { analysis }\end{array}$ & $\begin{array}{l}\text { FCFS } \\
\text { - CFS }\end{array}$ & Length & Width & Thick & $\begin{array}{l}\text { Scraper } \\
\text { Shape }\end{array}$ & EA & Motions & $\begin{array}{l}\text { Worked } \\
\text { Material }\end{array}$ \\
\hline 15 & 9029 & 90143 & & $\mathrm{C}$ & late PN & late PN & late PN & CFS & 5.4 & 5.6 & 1.5 & $\begin{array}{l}\text { round with } \\
\text { notch }\end{array}$ & $\mathrm{m}$, steep & $\begin{array}{c}\text { cut, } \\
\text { scrape }\end{array}$ & $\begin{array}{l}\text { meat, fresh } \\
\text { hide, bone }\end{array}$ \\
\hline 16 & 2009 & 20113 & & D & late PN & TS & late PN & FCFS & 5.3 & 7.5 & 1.1 & fan & $\begin{array}{l}\text { srp, m, } \\
\text { stp }\end{array}$ & $\begin{array}{l}\text { cut, } \\
\text { scrape }\end{array}$ & $\begin{array}{l}\text { meat,fresh } \\
\text { hide,bone? }\end{array}$ \\
\hline 17 & 3041 & 30125 & & $\mathrm{E}$ & EBA1b & EBA & Mixed & FCFS & 7.3 & 2.1 & 0.9 & elongated & $m$, steep & $\begin{array}{l}\text { scrape } \\
\text { (cut) }\end{array}$ & $\begin{array}{l}\text { hide } \\
\text { (ochre?), } \\
\text { meat }\end{array}$ \\
\hline 18 & 3043 & 30140 & & $\mathrm{E}$ & EBA1b & EBA & Mixed & FCFS & 7.8 & 3.8 & 1.2 & elongated & moderate & $\begin{array}{l}\text { cut, } \\
\text { scrape }\end{array}$ & $\begin{array}{l}\text { meat, fresh } \\
\text { hide, bone }\end{array}$ \\
\hline 19 & 3135 & 30750 & & $E$ & late PN & late PN & late PN & FCFS & 6.7 & 7.2 & 0.9 & $\begin{array}{c}\text { fan } \\
\text { (fragment) }\end{array}$ & m, steep & $\begin{array}{l}\text { cut, } \\
\text { scrape }\end{array}$ & $\begin{array}{l}\text { meat, fresh } \\
\text { hide, bone }\end{array}$ \\
\hline 20 & 3135 & 30816 & & $E$ & late PN & late PN & late PN & CFS & 4.9 & 4.4 & 0.9 & $\begin{array}{c}\text { oval } \\
\text { (fragment) }\end{array}$ & $\begin{array}{c}\text { m, sharp } \\
\text { stp }\end{array}$ & $\begin{array}{c}\text { cut, } \\
\text { scrape }\end{array}$ & $\begin{array}{l}\text { meat, fresh } \\
\text { hide, bone }\end{array}$ \\
\hline 21 & 7701 & 77010 & & $\mathrm{~N}$ & Mixed & TS & Mixed & CFS & 3.7 & 5.5 & 0.9 & $\begin{array}{l}\text { fan with } \\
\text { notch }\end{array}$ & m, steep & scrape & $\begin{array}{l}\text { fresh hide, } \\
\text { meat, bone }\end{array}$ \\
\hline 22 & 1468 & 13771 & & B & EBA1b & TS & Mixed & CFS & 5 & 3.6 & 0.5 & fragment & sharp & scrape & $\begin{array}{l}\text { fresh hide } \\
\text { (ochre?) }\end{array}$ \\
\hline 31 & 1976 & 17804 & & C & late PN & late PN & late PN & CFS & 6.2 & 6.9 & 1.6 & irregular & moderate & $\begin{array}{l}\text { cut? } \\
\text { scrape }\end{array}$ & $\begin{array}{l}\text { meat, fresh } \\
\text { hide, bone }\end{array}$ \\
\hline
\end{tabular}


Table 2. Ein Zippori fan scraper spalls microwear results. Abbreviations: Loc. = Locus, Buck. = Bucket; late PN = late Pottery Neolithic Wadi Rabbah, EBA = Early Bronze Age1b, cultural period based on stratigraphy and pottery analysis, TS = mixed surface; FCFS = flat cortex fan scraper with flat dorsal face, CFS = cortical fan scraper with convex dorsal face; $\mathrm{GL}=$ greatest length, $\mathrm{GW}=$ greatest width, $\mathrm{Th}=$ thickness in $\mathrm{mm}$; elong. = elongated, frag. $=$ fragment, irreg. $=$ irregular shapes; $\mathrm{m}=$ moderate, srp = sharp, stp = steep edge-angles.

\begin{tabular}{|c|c|c|c|c|c|c|c|c|c|c|c|c|c|c|c|}
\hline $\begin{array}{l}\text { MW } \\
\#\end{array}$ & Locus & Bucket & $\#$ & Area & $\begin{array}{l}\text { Cultural } \\
\text { Context }\end{array}$ & $\begin{array}{l}\text { Layer } \\
\text { according to } \\
\text { stratigraphy }\end{array}$ & $\begin{array}{l}\text { Period } \\
\text { according } \\
\text { to } \\
\text { pottery } \\
\text { analysis }\end{array}$ & Typology & Length & Width & Thick & $\begin{array}{l}\text { Edge } \\
\text { shape }\end{array}$ & EA & Motions & Worked material \\
\hline 2 & 1970 & 17940 & & $\mathrm{C}$ & late PN & late PN & Mixed & burin & 3.8 & 1.1 & 0.9 & convex & moderate & cut, scrape & $\begin{array}{l}\text { meat, fresh hide, } \\
\text { bone }\end{array}$ \\
\hline 3 & 2189 & 20541 & & $\mathrm{D}$ & EBA1b & EBA & Mixed & burin & 3.8 & 0.9 & 0.7 & straight & moderate & cut, scrape & $\begin{array}{l}\text { meat, fresh hide, } \\
\text { bone }\end{array}$ \\
\hline 4 & 2041 & 20195 & & $\mathrm{D}$ & EBA1b & EBA & EBA & other & 5.3 & 2.1 & 2.0 & convex & steep & scrape? & hide, meat? \\
\hline 5 & 3135 & 30816 & & $E$ & late PN & late PN & Mixed & burin & 4.5 & 1.3 & 1.1 & convex & moderate & scrape (cut?) & hide, meat (bone?) \\
\hline 6 & 3158 & 30857 & & $\mathrm{E}$ & late PN & late PN & late PN & other & 1.9 & 2.3 & 1.5 & straight & moderate & scrape & dry hide \\
\hline 8 & 6065 & 60353 & 2 & L & EBA1b & EBA & EBA & burin & 6.4 & 1 & 0.9 & convex & moderate & cut, scrape & $\begin{array}{l}\text { meat, fresh hide, } \\
\text { bone }\end{array}$ \\
\hline 9 & 6065 & 60353 & 3 & $\mathrm{~L}$ & EBA1b & EBA & EBA & 2nd burin & 4.8 & 0.8 & 0.7 & convex & steep & scrape & hide, meat \\
\hline 10 & 6084 & 60271 & & $\mathrm{~L}$ & EBA1b & EBA & EBA & other & 4.1 & 1.6 & 1.0 & straight & moderate & cut, scrape & $\begin{array}{l}\text { meat, fresh hide, } \\
\text { bone }\end{array}$ \\
\hline 13 & 3261 & 31425 & & $\mathrm{E}$ & late PN & late PN & Mixed & burin & 3.5 & 0.8 & 0.9 & convex & moderate & scrape (cut?) & fresh hide, meat \\
\hline
\end{tabular}




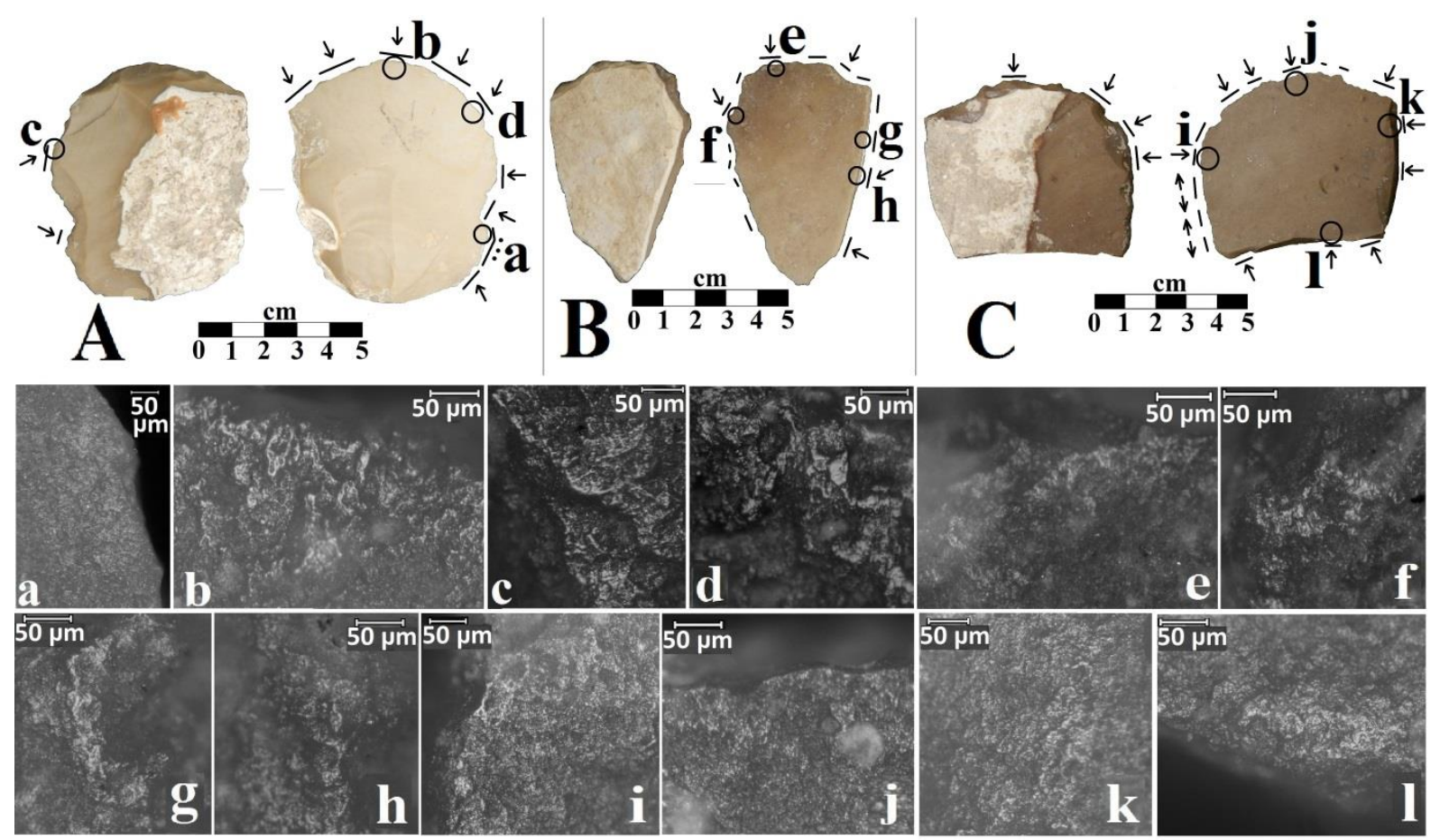

Figure 2. Fan scraper with prehension traces $(\mathbf{A}, \mathbf{a})$ and scrapers with steep edge-angles used to smooth bone and also to scrape bone to remove meat or periosteum $(\mathbf{A}, \mathbf{B}, \mathbf{C})$. Bright bone and rough meat polish shown on $\mathbf{b}-\mathbf{j}$, and $\mathbf{l}$. Fresh hide or meat wear traces in from edges $(\mathbf{k})$ are on $\mathbf{C}$ not well-developed. Fan scrapers were held in the hand and used for butchering and scraping meat off bones. Photomicrographs are 187.5x, scales are 50 microns. A. Dorsal (left) and ventral (right) faces of oval cortical fan scraper (CFS, MW\#5) from Area B, late Pottery Neolithic (PN) level, Locus 3538, Bucket15055. B. Triangular CFS fan scraper fragment (MW\#10) from Area C, late PN level, Locus 1976, Bucket17805. C: Flat FCFS fan scraper fragment with geometric incisions on cortex (MW\#19) from Area E, late PN level, Locus 3135, Bucket30750. White lines show extent of the usewear, line with dots: location of prehension traces, arrows show orientations of microwear polishes and striations, circles: locations of microphotographs a-l.

Microwear on nine utilized fan scraper spalls was similar to usewear on fan scrapers (Tables 1 and 2). Most spalls were struck from tools used to cut and scrape meat, fresh, hide, and bone (Figure 3).

When the functions of cortical fan scrapers $(n=14)$ and flat cortex fan scrapers $(n=6)$ used for four of the five identified tasks summarized in Figure 1 are compared (only fan scraper spalls had wear from cutting fresh or dry hide and meat), there also is no difference in frequencies of tools used for different tasks (Chi-square $=1.75 ; \mathrm{T}(0.001)=16.27,3 \mathrm{df})$.

\subsection{Edge-angles and notches on fan scrapers and fan scraper spalls}

A goniometer was used to measure 62 edge angles on 22 utilized fan scrapers and nine fan scraper spalls (Table 3). Most had moderate edge-angles of $45^{\circ}-74^{\circ}(55 \%), 27 \%$ had steep edge-angles $\left(75^{\circ}-90^{\circ}\right)$, and $18 \%$ had sharp edge angles $\left(30^{\circ}-44^{\circ}\right.$. Almost all steep edges (94\%) were used for scraping (Table 3). They were on fan scrapers used for skinning animals and scraping hides or bones (either to remove meat from bones, or to make bone tools). For moderate edges, $67 \%$ were used for scraping, $24 \%$ for cutting, and $9 \%$ for both. Most sharp edges $(45 \%)$ were also used for scraping, but 36\% were used for cutting and $18 \%$ for both. Half of the 22 utilized fan scrapers had edges with different edge-angles. Six had steep edges used for scraping and sharp or moderate edges used for cutting. Notches on three scrapers (MW\#10, 15, 21) were used to scrape meat off of bone or to make bone tools (Tables 1, Figure $2 \mathrm{~A}$ and $\mathrm{B}$ ). Variation in edge-angles on the fan scrapers made each tool suitable for 
cutting, scraping, and sawing, and supports the interpretation of Quintero et al. (2002: 46) that fan scrapers were multi-purpose tools.
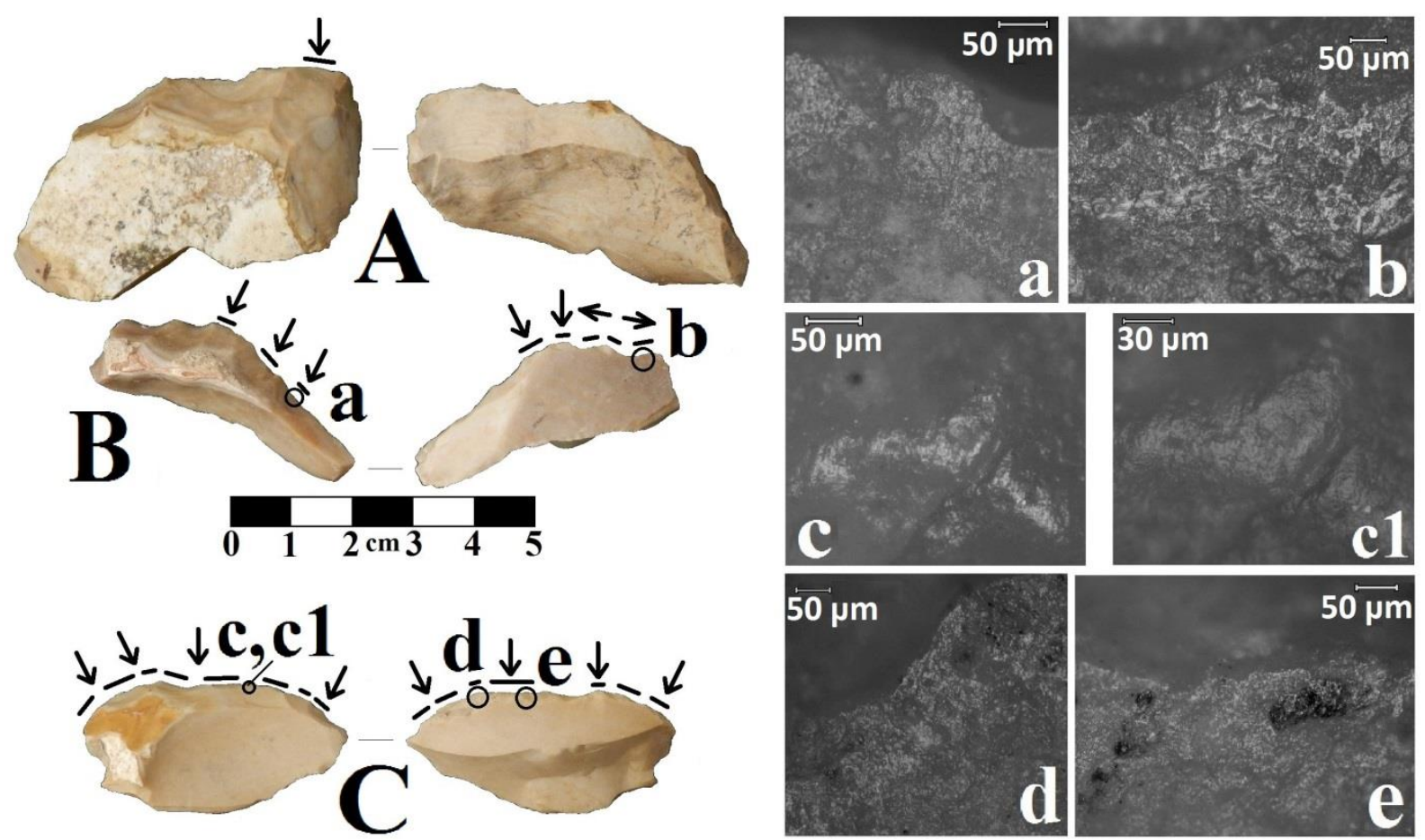

Figure 3. Three spalls (A, B, C) removed from fan scrapers used to skin and butcher animals. a, d, e: edgerounding and rough "greasy" hide or meat polish (black spots on $\mathbf{d}$ and $\mathbf{e}$ ). $\mathbf{c}$ and $\mathbf{c 1}$ : Heavy edge-rounding and microwear from dry hide. Microwear on A weakly-developed. All photomicrographs except c1 are at 187.5x, with scales that are 50 microns. The scale on $\mathbf{c 1}$ is 30 microns, and magnification is $375 x$. A: Thick convexedged spall of banded flint (MW\#4) Area D, Early Bronze Age (EBA) layer, Locus 2041, Bucket 20195. B: Lustrous convex fan scraper spall (MW\#5) with patina, Area E, late Pottery Neolithic (PN) layer, Locus 3135, Bucket 30816. C: Lustrous straight-edged fan scraper spall (MW\#6) with patina, Area E, late PN layer, Locus 3158, Bucket 30857.

Table 3. Ein Zippori fan scrapers: edge-angles and tool motion. Abbreviations: N - number, Both - both cutting and scraping.

\begin{tabular}{|c|c|c|c|c|c|c|c|c|c|}
\hline \multirow{3}{*}{ Edge classification } & \multirow{3}{*}{ Angles } & \multicolumn{6}{|c|}{ Dominant motion } & \multirow{2}{*}{\multicolumn{2}{|c|}{ Total }} \\
\hline & & \multicolumn{2}{|c|}{ Scraping } & \multicolumn{2}{|c|}{ Cutting, sawing } & \multicolumn{2}{|c|}{ Both } & & \\
\hline & & $\mathbf{N}$ & $\%$ & $\mathbf{N}$ & $\%$ & $\mathbf{N}$ & $\%$ & $\mathbf{N}$ & $\%$ \\
\hline Steep & $90-75$ & 15 & $94 \%$ & 1 & $6 \%$ & 0 & $0 \%$ & 16 & $27 \%$ \\
\hline Moderate & $74-45$ & 22 & $67 \%$ & 8 & $24 \%$ & 3 & $9 \%$ & 33 & $55 \%$ \\
\hline Sharp & $44-30$ & 5 & $45 \%$ & 4 & $36 \%$ & 2 & $18 \%$ & 11 & $18 \%$ \\
\hline Total & & 44 & $70 \%$ & 13 & $22 \%$ & 5 & $8 \%$ & 60 & $100 \%$ \\
\hline
\end{tabular}

\subsection{Bone scrapers}

Three fan scrapers (two CFS, MW\#5, 10, and one FCFS, MW\#11) with steep edges were only used to smooth bone and scrape bone to remove meat or periosteum (Figure $2 \mathrm{~A}$ and $\mathrm{B}$ ). Another fan scraper (FCFS, MW\#19, Figure 2 C) also had fresh hide or meat cutting traces on its moderate lateral edge, but that microwear was not well-developed. It also had geometric incisions (see fig. 12 and discussion in Zutovski et al. 2016). 


\subsection{Skinning and butchering tools}

Most late PN and EBA fan scrapers from Ein Zippori, including CFS and FCFS fanshaped, elongated, oval, round, and irregular types, were hand-held tools for skinning and butchering animals (Tables 1). McConaughy (1979) and Rowan and Levy (1991) also found evidence for these tasks on fan scrapers from Bâb edh-Dhrâ and Shiqmim. Edges of two Ein Zippori CFS fan scrapers used for skinning and butchering (MW\#15, 22) (Figure 4) may have been notched to scrape meat off of bones, or for bone tool manufacturing. On fan scrapers and spalls with sharp and moderate edges, microwear from cutting and scraping fresh hide or meat is common (Tables 1 and 2), and sometimes evidence for sawing bones is visible (MW\#3, 6) (Figure 5).
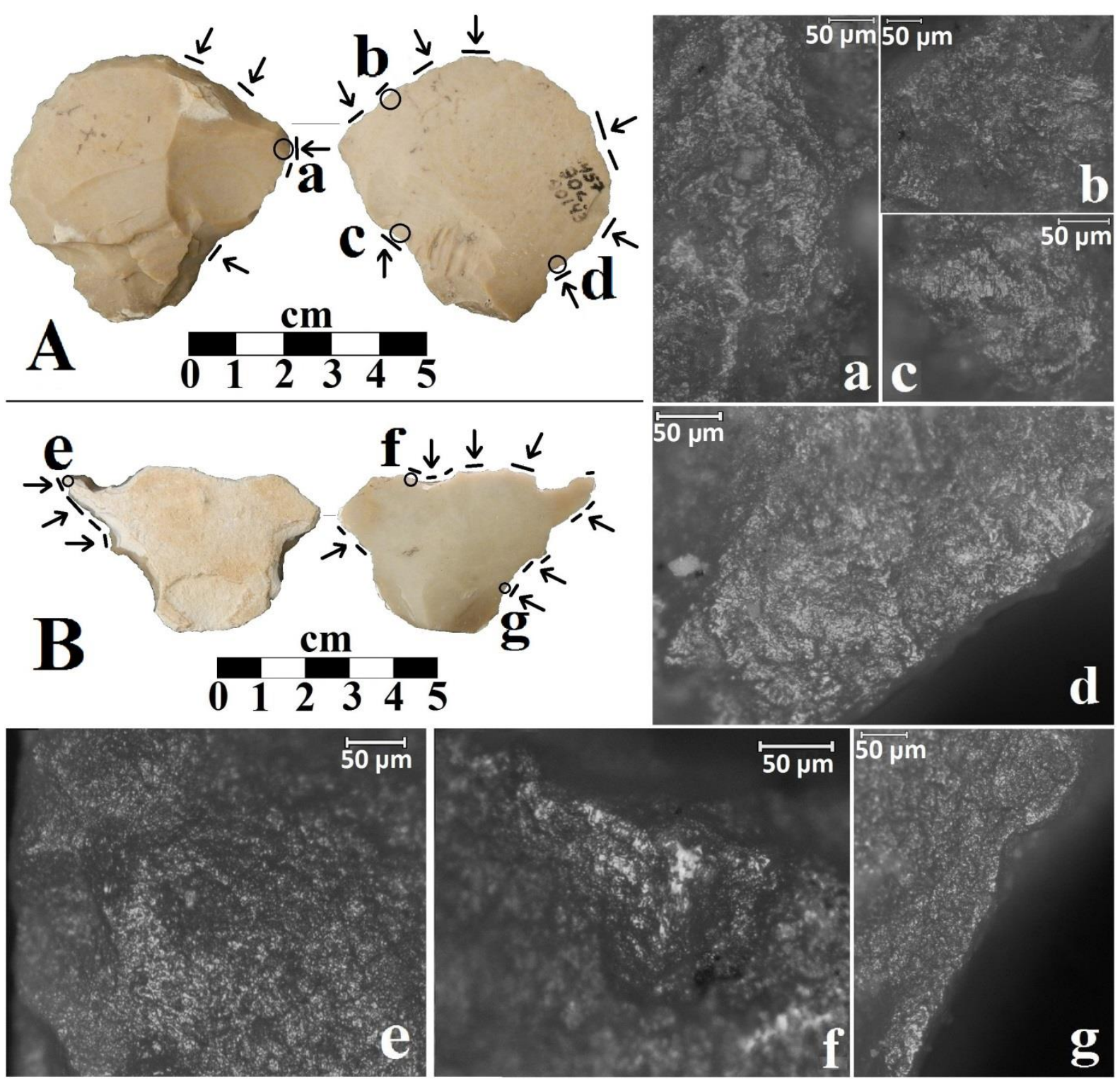

Figure 4. CFS fan scrapers (A, B) used for butchering and skinning that were notched and used to scrape meat off bones or manufacture bone tools. a, b, e: rough "greasy" meat or fresh hide polish on edges used for butchering and skinning. $\mathbf{c}, \mathbf{d}$, rough "greasy" polish in notches of A. f, g: bright bone polish in notches of B. A: Dorsal (left) and ventral (right) faces of MW\#15, round fan scraper, Area C, late PN layer, Locus 9029, Bucket 90143. B. fan-shaped scraper (MW\#21, Area N, EBA layer, Locus 7701, Bucket77010. White lines show extent of usewear, arrows show orientation of microwear polishes and striations, circles show locations of microphotographs a-g. All photomicrographs are $187.5 \mathrm{x}$, scales are 50 microns. 

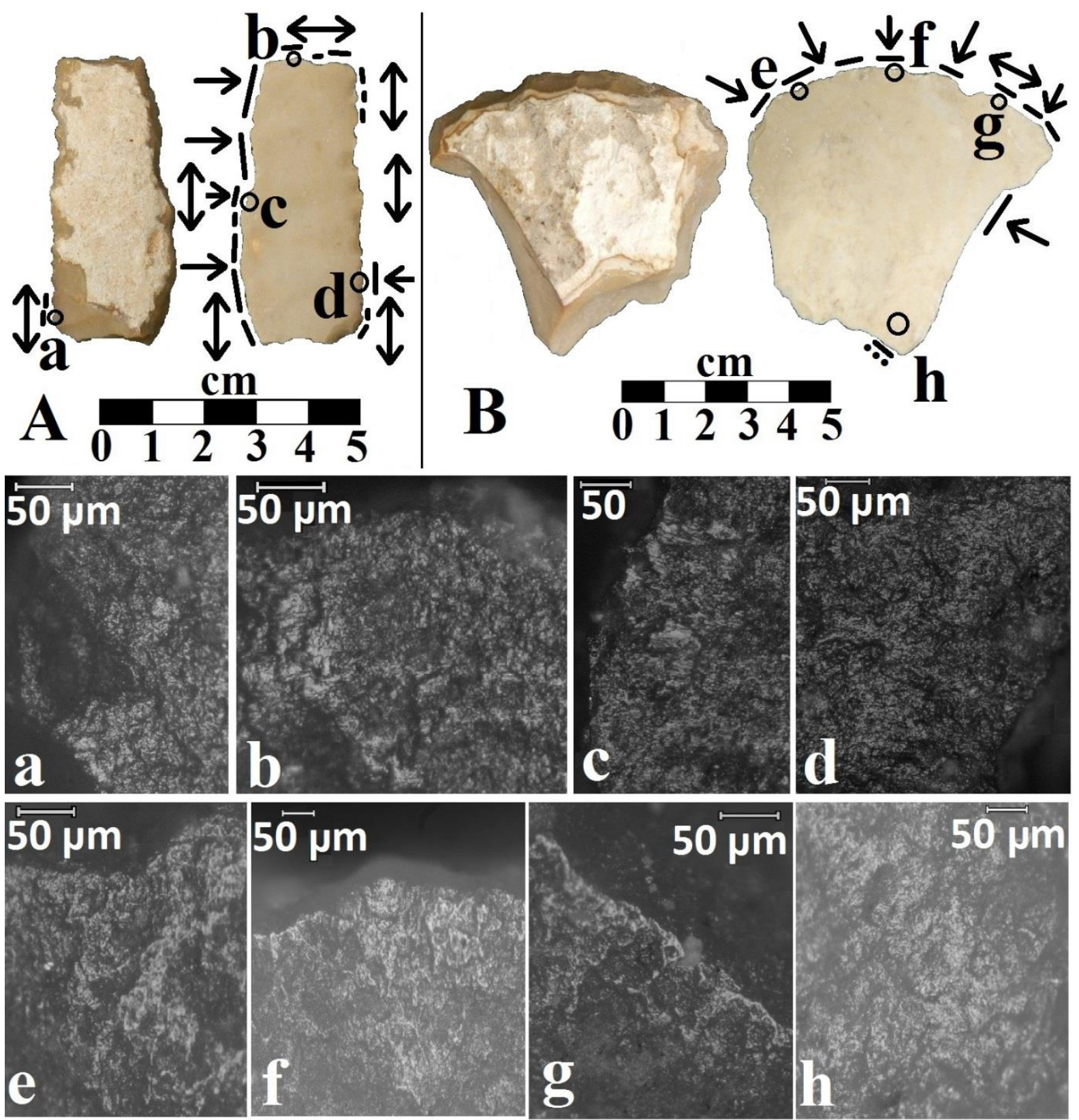

Figure 5. Fan scrapers (A, B) used to saw, cut, and scrape bone, meat, and fresh hide. a: bright bone and rough "greasy" fresh hide or meat cutting microwear. c, d, e, f: bright bone and rough "greasy" fresh hide or meat scraping wear. b, g: bright bone with striations and rough meat or hide wear from sawing. $\mathbf{h}$ : prehension traces. Sharp edges (a, d) used for cutting and scraping, moderate edges (b, c, e-g) used for scraping and sawing. A: Dorsal (left) and ventral (right) faces of elongated CFS fan scraper (MW\#3) Area B, EBA layer, Locus 1427 , Bucket 13471(\#2). B: Fan scraper fragment (MW\#6) Area B, late PN layer, Locus 3561, Bucket 15133. White lines: extent of usewear, line with dots: prehension traces, arrows show orientation of microwear polishes and striations, Circles: locations of microphotographs a-h. All photomicrographs $187.5 x$, scales are 50 microns.

Usewear traces on fan scrapers from Ein Zippori used for skinning and butchering are similar to microwear on experimental replicas used to butcher a deer and scrape fresh deer bone. (Figure 6 a1, e1). The experimental cutting and scraping usewear is compared to microwear on Ein Zippori fan scrapers in Figure 5. Both fan scrapers from Ein Zippori seem to have been used to scrape and remove fresh hide or meat with their steep edges (Figure 6 $\mathrm{b}, \mathrm{d}, \mathrm{e}, \mathrm{f}$ ), and cut fresh hide or meat with their sharp edges (Figure 6 a,c,g) 


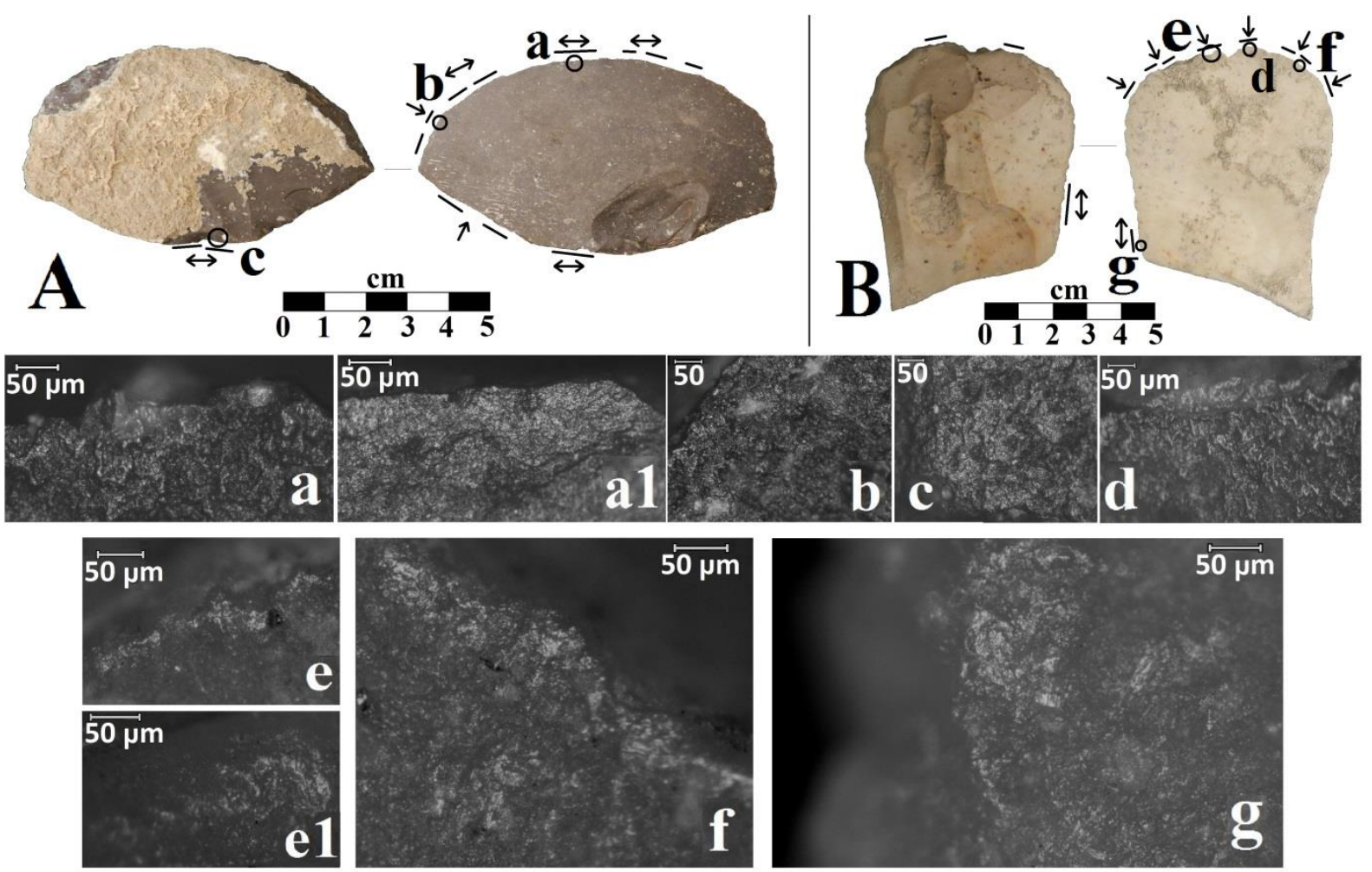

Figure 6. Microwear on Ein Zippori fan scrapers (A, B) and experimental deer butchering and fresh bone scraping tools compared. a: bright bone and rough "greasy" meat or fresh hide polish on distal edge of A. a1: Bright bone and rough "greasy" meat polish on experimental flint blade used to butcher a deer. e: bright bone polish and short striations along left distal edge of B. e1: bright bone polish and short striations along edge of experimental flint tool used to scrape fresh deer bone. b, d, f: bright bone and rough "greasy" meat or hide scraping microwear. c, g: bright bone and rough meat or hide cutting wear. A: Dorsal (left) and ventral (right) faces of FCFS fan-shaped scraper (MW\#16), Area D, late PN, Locus 2009, Bucket 20113. B. Oval CFS fan scraper fragment (MW\#9) Area C, late PN, Locus 1944, Bucket 17825. White lines: extent of usewear, arrows show the orientation of microwear polishes and striations, circles: locations of microphotographs a-g. Photomicrographs $187.5 x$, scales are 50 microns.

\subsection{Scraping or cutting fresh hides with thick fat layers}

Bright and glossy microwear polish on an EBA FCFS elongated fan scraper (MW\#17) and an oval EBA CFS scraper (MW\#22) (Figure 7A) was similar to the wear traces UngerHamilton (1991) described. While she suggested that this was produced by scraping reeds, heavy edge-rounding and deep striations on the elongated Ein Zippori scraper do not match reed-cutting wear traces (Figure 7a). The severe edge-rounding, striations, and bright hide polish on the two scrapers (Figure 7) are similar to microwear traces on an experimental scraper van Gijn (1990: fig. 13b) used on fresh hide with thick subcutaneous fat layers that was treated with powdered ochre (Figure 7 a1). This bright hide polish is similar to plant or reed microwear, and also the unique microwear on European Neolithic burin spalls ("Frits") that may have formed when hides treated with a plant-based compound were cleaned (Sliva \& Keeley 1994), but it has significant edge-rounding and numerous striations. The wear on both EBA Ein Zippori scrapers (Figure 7) is more like the microwear produced when abrasives are added to help remove fat from hides (van Gijn 1990: 27, 30, fig. 13b; 2010: fig. 4.6d). 

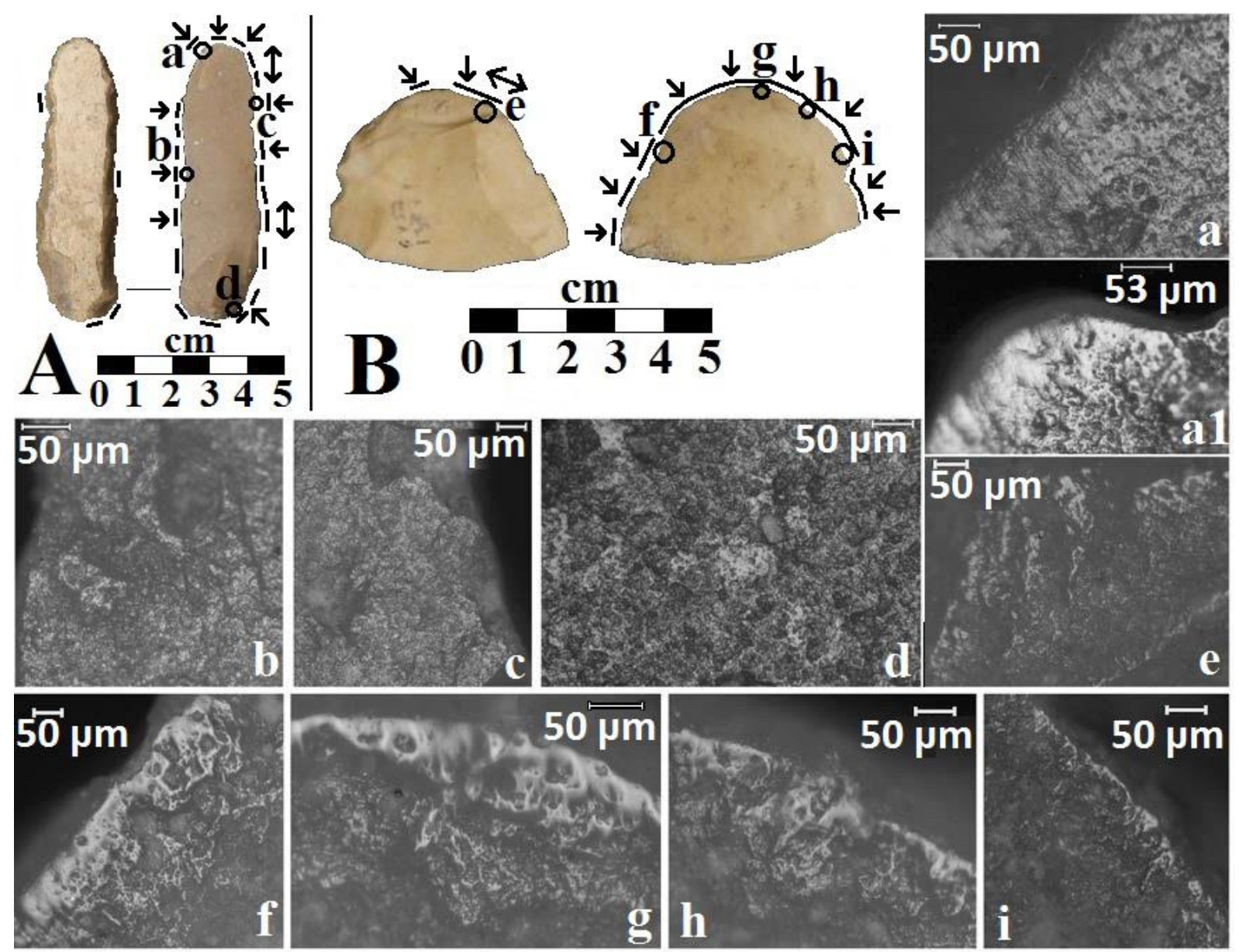

Figure 7. EBA fan scrapers (A, B) from Ein Zippori with microwear from scraping and cutting hides with thick fat layers. a, d: severe edge-rounding, striations, and well-developed, bright hide polish on A. a1: wear traces on experimental scraper van Gijn (1990: fig. 13b) used on hide with powdered ochre and liver. b, c: duller rough "greasy" fresh hide or meat wear on A. e-i: "hide-ochre" polish similar to a1 on B. A: Dorsal (left) and ventral (right) faces of elongated FCFS fan scraper (MW\#17) Area E, EBA layer, Locus 3041, Bucket 30125. B: Oval CFS fan scraper fragment (MW\#22) Area B, EBA layer, Locus 1468, Bucket 13771. White lines: extent of usewear, arrows show orientation of microwear polishes and striations, circles: locations of microphotographs ai. Photomicrographs are $187.5 \mathrm{x}$ and scales are 50 microns, except for a1 where scale is 53 microns, and magnification is $200 x$.

\subsubsection{Heat-treated fan scraper}

The only heat-treated fan scraper in the Ein Zippori microwear sample was a lustrous burgundy-colored oval CFS fan scraper (MW\#12). Only one sharp edge was utilized. Effects of thermal treatment can been seen in the heat-cracked cortex that covers the dorsal face (Figure 8).

Heat treatment produced a lustrous sheen, but there was extensive fresh hide or meat microwear and bright bone polish that was similar to usewear traces on an experimental tool used to skin and butcher a deer (Figure 8a2, c1). One portion of the used edge also had greasy "feathered" meat or fresh hide polish (Figure 8a1) that was similar to wear on a tool replica made of lustrous flint that was used to butcher a deer (Figure 8c2). It was not like the meat or hide microwear usually produced when deer are skinned and butchered (Keeley 1980: Plates 36,41-48; van Gin 1990: fig. 13a, 2010: fig 4.2b; Yerkes 1987: Plate 4a,b). While the lustrous surface of the replica and heat-treated Ein Zippori fan scraper could have produced the feathered appearance, the CFS scraper may have been used on animals with thick fat layers. 
Further experiments and contextual investigations are need to support the idea that fan scrapers like this one were used to sacrifice "fatted calves" or other young animals, however.
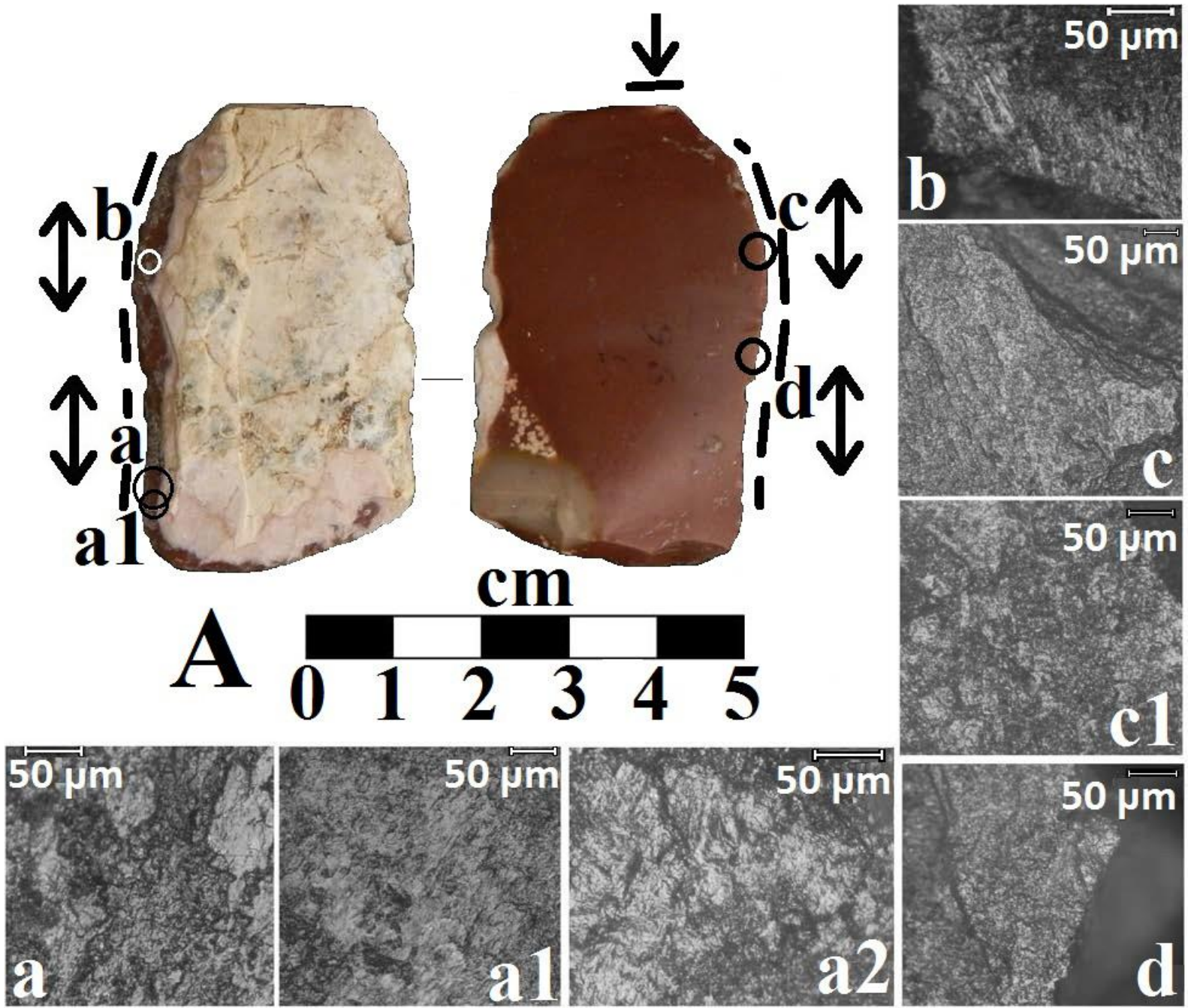

Figure 8. Late PN Heat-treated CFS fan scraper (A) from Ein Zippori used for skinning and butchering animals, possibly with thick fat layers, but without added abrasives. a, a1: rough "greasy" and "feathered" meat or fresh hide microwear on A. a2: Rough "greasy" polish on lustrous flint tool replica used to skin and butcher a deer. b, d: streaks of bright bone, striations, and rough hide or meat polish on $\mathbf{A}$. c: rougher meat or fresh hide polish on A. c1: meat or fresh hide polish on another edge of tool replica used to skin and butcher a deer. A: Dorsal (left) and ventral (right) faces of heat-treated oval CFS fan scraper (MW\#12) Area C, late PN layer, Locus 1996, Bucket 90039. White lines: extent of usewear, arrows show orientation of polishes and striations, circles: locations of microphotographs $\mathbf{a}, \mathbf{a 1}, \mathbf{b}, \mathbf{c}, \mathbf{d}$. All photomicrographs are $187.5 \mathrm{x}$, all scales are 50 microns.

\section{Discussion and conclusions}

Microwear analysis was conducted to test hypotheses about functions of fan scrapers and their role in late prehistoric Levantine economy and ritual. Ein Zippori fan scrapers were not used as plant processing or reed cutting tools (Baird 2001: 649; Unger-Hamilton 1991), and there is little support for McConaughy's (1980: 53-59) interpretation that fan scrapers were used as sacrificial knives. Three Ein Zippori fan scrapers were probably used to cut scrape hides from animals with thick fat layers, but this does not prove that they were ritual tools.

Microwear results did show that Ein Zippori fan scrapers were hand-held multi-purpose tools, consistent with Quintero et al.'s interpretation (2002: 46). Different working edges on many fan scrapers had steep, moderate, or sharp edge-angles, and some had been notched. Edges on the same scraper were used for cutting, scraping, sawing, or cutting and scraping 
(Table 3). Most fan scrapers from Ein Zippori were skinning and butchering tools, and they were also used for processing hides, and scraping bone (Tables 1 and 2). Rather than using several implements in tool kit, Ein Zippori residents often used a single fan scraper for the entire skinning and butchering process.

\subsection{Secondary products of domestication}

Microwear on fan scrapers, scraper fragments, and spalls from late PN and EBA contexts at Ein Zippori were not significantly different. Why did this distinctive scraper appear in the Late PN and continue to be used during the CHG and EBA periods? The appearance and the continued use of fan scrapers may be related to agricultural intensification (e.g., larger-sized herds and flocks, stock breeding, and the use of secondary products of domestication like milk, wool, dung, and animal power) that began in the late PN and became even more marked in the EBA. Andrew Sherratt $(1997 \mathrm{a} ; 1997 \mathrm{~b})$ argued that the "secondary products revolution" (SPR) was a Bronze Age phenomenon - and fan scrapers have been described as a diagnostic tool type in lithic assemblages from EBA sites throughout the southern Levant. However, the shift to an emphasis on secondary products seems to have been gradual (Grigson 2000; Halstead and Issakidou 2011). Evidence for this gradual shift is reflected in the technology and economy of late PN and CHG societies.

The PN economy was based on domesticated animals and grain crops that had been introduced to the Levant in earlier periods (Davis, 2012: 1259; Gopher, 2012: 1553). A decrease in remains of game and numbers of arrowheads, and an increase in remains of domesticated animals, is evident at PN sites (Davis, 2012: 1258, table 31.1; Gopher, 2012: 1553). However, domesticated animals were still exploited mainly for meat and hide rather than milk and wool (Davis, 2012: 1269; Grigson, 2000). It was argued that changes in sheep or goat ratios, and ages at slaughter may provide some insights about the SPR in the Levant. An increase in sheep and a decline in goat remains may indicate the use of sheep wool for textiles, while mortality profiles with an increase in older adults may reflect an emphasis on milk and wool exploitation, rather than raising animals just for meat and hides (Davis 2012: 1269; Grigson 2000). Late PN sites still exhibit a pre-SPR pattern where goats are more common than sheep, and there are younger ages at death for adults (Davis 2012: 1269; Grigson 2000). A growing emphasis on sheep husbandry was detected in later periods beginning with the sixth, and especially the fifth, millennia cal BP, and was linked to a wool textile industry (Vila and Helmer 2014; Wilkinson et al. 2011 and references therein). Although a few ceramic churns were found in Late PN sites, secondary products exploitation was more limited during the Late PN (Rosen, 2012: 1351) than it was in the later CHG. Fan scrapers are associated with agricultural intensification during the Bronze Age, but at Ein Zippori (and other sites) appear during the late PN. This suggests that use of secondary animal products had begun at that time.

Experiments by Barket and Bell (2011) demonstrated that fan scrapers could have been used by ancient sheep and goat herders living in desert regions as wool-shearing knives, as Henry (1995: 372-373) and Bennett et al. (1989) had suggested. However microwear on the edges of replica fan scrapers that Barket and Bell illustrated (2011: fig. 5) is similar to microwear on the edges of experimental tools used on other soft materials (hide, meat, and plants). Their study did not confirm the wool-shearing hypothesis and does not provide support for a shift to the use of some secondary products by the dessert groups. Some of the brighter hide polish on the elongated fan scraper and the oval fan scraper fragment (Figure 6) from EBA levels at Ein Zippori may be the result of shaving lanolin-rich wools off of sheep. However, the overall appearance of the microwear on the two scrapers resembles usewear on experimental flint tools used to skin and butcher animal with thick fat layers, when some 
abrasives and tanning agents were added (van Gijn 1990, 2010). Other microwear on the elongated fan scraper (Figure 6A), and most fan scrapers and fan scraper spalls in the Ein Zippori microwear sample resemble microwear that formed on experimental flint tools used to skin and butcher "leaner" white-tail deer, not animals with thicker fat layers, or from wool shearing.

In spite of the typological variation in fan scraper shapes, and the variation the in edgeangles on their different working edges, they are a distinctive recognizable type of flaked stone tool. They were made on relatively flat and thin cortical flakes and could be easily resharpend and repaired (Quintero et al. 2002: 17; Zutovski et al. 2016). Their innovative design for multiple animal processing tasks seems to have been associated with a demand for more efficient skinning and butchering tools at a time when the size of animal herds and flocks increased. If more livestock had to be skinned and butchered, fan scrapers may have made this task easier. It has long been thought that they were a specialized tool, and the fact that they did not replace "typical" side and end scrapers in late PN, CHG, and EBA lithic assemblages confirms this. However, questions remain about how fan scrapers relate to the emergence of specialized stock raising and emphasis on secondary products of domestication during the late PN. The results of the microwear analysis show that intensive skinning and butchering of animals probably occurred at sites, or areas within sites, where large numbers of fan scrapers are found. If these sites date to the late PN period, this could be taken as evidence for specialization and agricultural intensification. Wool-shearing and ritual activities could also have occurred at or near these locations, but more evidence is needed to demonstrate that this was so.

\subsection{Summary and Conclusions}

A representative sample of 31 fan scrapers and 14 fan scraper spalls from late Pottery Neolithic (PN) and Early Bronze Age (EBA) contexts at the Ein Zippori site were selected for microwear analysis. Identifiable wear traces were found on $22(71 \%)$ of the fan scrapers and nine $(64 \%)$ of the spalls in the sample. Two fan scrapers only had weak generic microwear, but motions and worked materials could be identified on the others. Microwear was visible on several different edges of the scrapers. Edge-angles on the same scrapers often were variable: steep, moderate, or sharp. The majority of the utilized fan scrapers and spalls in the Ein Zippori sample had microwear that showed that they were used for both cutting (with sharper edges) and scraping (with steeper edges), and sometimes also for sawing, fresh hide, meat, and bone. Other scrapers and spalls had dry hide and bone scraping traces on their edges. Fan scrapers were not hafted, and the cortex on their dorsal face may have made it easier to hold on to them when they were used to skin and butcher animals. There was no significant difference in the functions of fan scrapers from late PN and EBA levels. In spite of the variation in the shape and edge-angles on the fan scrapers and spalls, these distinctive tools were used for skinning and butchering animals, and may be associated with intensified and specialized animal processing that began in the late PN and continued into the EBA when there was also greater emphasis on secondary animal products. While use of fan scrapers for reed-cutting, wool-shearing, and ritual activities had been proposed, there was little, if any, support for this in the microwear results.

\section{References}

Baird, D. 2001, The Analysis of Chipped Stone in Jordanian Archaeology. In: The Archaeology of Jordan (MacDonald, B., Adams, R., \& Bienkowski, P., Eds.), Sheffield Academic Press, Sheffield: p.693-706. URI: http://repository.liv.ac.uk/1057367 
Bankirer, R. Y., \& Marder, O. 2003, The flints from Tel Gat-Hefer. 'Atiqot, 44:139-142.

Barkai, R. 2004, The Chalcolithic Lithic Assemblage. In: Giv'at Ha-Oranim, A Chalcolithic Site (Scheftelowitz, N., \& Oren, R., Eds), Salvage Excavation Reports Vol. 1, Sonia and Marco Nadler Institute of Archaeology, Tel Aviv University, Tel Aviv: p.87-109. URL: http://archaeology.tau.ac.il/arch_files/info/ran_barkai/III.pdf

Barkai, R., \& Gopher, A. 2012, Flint assemblages from Nahal Zehora II: Techno-typological changes during the PN. In: Village Communities of the Pottery Neolithic Period in the Menashe Hills, Israel: Archaeological Investigations at the sites of Nahal Zehora (Gopher, A. Ed.), Emery and Claire Yass Publications in Archaeology Vol. 29, Institute of Archaeology, Tel Aviv University, Tel Aviv: p. 757-869.

Barket, T.M., \& Bell, C.A. 2011, Tabular Scrapers: Function Revisited. Near Eastern Archaeology, 74(1):56-59. doi:10.5615/neareastarch.74.1.0056

Bar-Yosef, O., Belfer, A., Goren, A., \& Smith, P. 1977, The nawamis near 'Ein Huderah (Eastern Sinai). Israel Exploration Journal, 27: 65-88.

Beit-Arieh, I., (Ed.), 2003, Archaeology of Sinai. The Ophir Expedition. Emery and Claire Yass Publications in Archaeology Vol. 21, Institute of Archaeology, Tel Aviv University, Tel Aviv, 454 p. URL: http://archaeology.tau.ac.il/?page_id=1440

Bennett, W.J. Jr., Sollberger, J.B., \& Gettys, A.F. 1989, Flint Tools. In: Tell el-Hesi: the Persian Period (Stratum V) (Bennett, W.J., Blakely, J.A., O'Connell, K.G., Horton, F.L., \& Coogan, M.D., Eds.), Excavation Reports: Joint Archaeological Expedition to Tell el-Hesi Vol. 3. Eisenbrauns, Winona Lake, IN: p. 231-326.

van den Brink, E., Horwitz, L.K., Khalaily, H., Liphschitz, N., Mienis, K., \& Nagar, Y. 2004, A Chalcolithic Dwelling and Burial Cave at Horvat Castra. Israel Exploration Journal, 54(2): 129-153. URL: http://www.jstor.org/stable/27927073

Crowfoot-Payne, J. 1983, The flint industries of Jericho. In: Excavations at Jericho Vol. V (Kenyon, K.A., \& T. A. Holland, T.A., Eds.), British School of Archaeology in Jerusalem, London: p. 622-759.

Davis, S.J. M. 1987, The Archaeology of Animals. Yale University Press, New Haven, 224 p.

Davis, S.J.M. 2012, Animal Remains at the Nahal Zehora Sites. In: Village Communities of the Pottery Neolithic Period in the Menashe Hills, Israel: Archaeological Investigations at the Sites of Nahal Zehora (Gopher, A., Ed.), Emery and Claire Yass Publications in Archaeology Vol. 29, Institute of Archaeology, Tel Aviv University, Tel Aviv: p. 12581320.

Elliott, C. 1977, The Religious Beliefs of the Ghassulians, 4000-3100 B.C. Palestine Exploration Quarterly, 109:3-25. doi:10.1179/peq.1977.109.1.3

Fujii, S. 1999, Qa` Abu Tulayha West: An Interim Report of the 1998 Season. Annual of the Department of Antiquities of Jordan, 43: 69-89.

van Gijn A.L. 1990, The Wear and Tear of Flint: Principles of Microwear Analysis Applied to Dutch Neolithic Assemblages. Analecta Praehistorica Leidensia Vol. 22, University of Leiden, Leiden, $182 \mathrm{p}$.

van Gijn, A. 2010, Flint in Focus: Lithic Biographies in the Neolithic and Bronze Age. Sidestone Press, Leiden, 289 p. 
Gopher, A. 1989, The flint assemblages of Munhata (Israel), final report. Paris: Les Cahiers du Centre de Recherche Francais de Jerusalem Vol. 4, Association Paleorient, Paris, $162 \mathrm{p}$.

Gopher, A. 2012, The Pottery Neolithic in the Southern Levant - A Second Neolithic Revolution. In: Village Communities of the Pottery Neolithic Period in the Menashe Hills, Israel: Archaeological Investigations at the sites of Nahal Zehora (Gopher, A. Ed.), Emery and Claire Yass Publications in Archaeology Vol. 29, Institute of Archaeology, Tel Aviv University, Tel Aviv: p. 1525-1611.

Greenhut, Z. 1989, יהודה במדבר חפירות. In: Excavations in the Judean Desert (Bar-Adon, P., Ed.), 'Atīqōt [Hebrew Series] Vol. 9, Atīqōt, Jerusalem: p. 60-77. (in Hebrew) ("Mispeh Shalem - Flint Tools")

Grigson, C. 2000, The Secondary Products Revolution? Changes in Animal Management from the Fourth to the Fifth Millennium at Arjoune, Syria. In: Archaeolozoology of the Near East IV (Mashkour, M., Choyke, A.M., Buitenhuis, H., \& Poplin, F., Eds.), Oxbow Books, Oxford: p. 12-28.

Halstead, P., \& Isaakidou, V. 2011, Revolutionary Secondary Products: the Development and Significance of Milking, Animal-Traction and Wool-Gathering in Later Prehistoric Europe and the Near East. In: Interweaving Worlds: Systemic Interactions in Eurasia $7^{\text {th }}-1^{\text {st }}$ Millennia B.C. (Wilkinson, T.C., Sherratt, S. \& Bennet, J., Eds.), Oxbow Books, Oxford: p. 61-76.

Henry, D. O. 1995, Prehistoric Cultural Ecology and Evolution: Insights from Southern Jordan. Plenum Press, New York, 466 p.

Juel Jensen, H. 1988, Functional Analysis of Prehistoric Flint Tools by High-Power Microscopy: a Review of West European Research. Journal of World Prehistory, 2: 53 88. doi:10.1007/BF00975122

Kaplan, J. 1969, Ein el Jarba: Chalcolithic remains in the plain of Eshdraelon. Bulletin of the American Schools of Oriental Research, 194:2-39. doi:10.2307/1356425

Keeley, L. H. 1980, Experimental Determination of Stone Tool Uses: A Microwear Analysis. University of Chicago Press, Chicago, 226 p.

URL: http://press.uchicago.edu/ucp/books/book/chicago/E/bo3615551.html

Khalaily, H. 2011, Nahal Yarmut: A Late Pottery Neolithic Site of the Wadi Rabah culture, south of Nahal Soreq. Atiqot, 67: 1-29.

Khalaily, H., \& Nagorsky, A. 2013, Tel Hanan: A Site of the Wadi Rabah Culture East of Haifa. Atiqot, 73:1-17.

Levy, T.E., \& Alon, D. 1985, Shiqmim: A Chalcolithic village and mortuary centre in the northern Negev. Paléorient, 11(1):71-83. doi:10.3406/paleo.1985.4362

Longo, L., \& Skakun, N., (Eds.), 2008, "Prehistoric Technology" 40 Years Later: Functional Studies and the Russian Legacy. Oxford, BAR International Series Vol. S1783, Oxford: Oxbow, Oxford, $559 \mathrm{p}$.

Mallon, R.P.A. 1932, Les Fouilles de Teleilat Ghassul. Syria, 13: 334-344. doi:10.3406/syria.1932.3650

Mallon, A., Koeppel, R., \& Neuville, R. 1934, Teleilat Ghassul I, 1929-32. Pontifical Biblical Institute, Rome, $193 \mathrm{p}$. 
Marder, O., Braun, E., \& Milevski, I. 1995, The Flint Assemblage of Lower Horvat' Illin : Some Technical and Economic Considerations. 'Atiqot, 27: 63-93.

McConaughy, M.A. 1979, Formal and Functional Analyses of the Chipped Stone Tools from Bâb edh-Dhrâ, Jordan. Ph.D. dissertation at the Department of Anthropology, University of Pittsburgh, Pittsburgh, 431 p.

McConaughy, M.A. 1980, F. Chipped Stone Tools. In: Preliminary Report of the 1979 Expedition to the Dead Sea Plain, Jordan (Rast, W.E., \& Schaub, R.T., Eds.), Bulletin of the American Schools of Oriental Research, 240: 53-59. doi:10.2307/1356536

Milevski, I., Liran, R., \& Getzov, N. 2014, The Early Bronze Age town of Ein Zippori in the Galilee (Israel). Antiquity, 88(339): 2-5.

URL: http://antiquity.ac.uk/projgall/milevski339/

Müller-Neuhof, B. 2006, Tabular scraper quarry sites in the Wadi Ar-Ruwayshid region (N/E Jordan). Annual of the Department of Antiquities of Jordan, 50: 373-383.

Noy, T. 1998, The flint artifacts. In: The Chalcolithic culture of the Golan (Epstein, C., Ed.), Israel Antiquity Authority (IAA) Reports Vol. 4, Jerusalem: p. 269-332.

Quintero, L.A., Wilke, P.J., \& Rollefson, G.O. 2002, From Flint Mine to Fan Scraper: The Late Prehistoric Jafr Industrial Complex. Bulletin of the American Schools of Oriental Research, 327: 17-48. doi:10.2307/1357856

Rosen, S.A. 1983, Tabular Scraper Trade: a Model of Material Cultural Dispersion. Bulletin of the American Schools of Oriental Research, 249: 79-86. doi:10.2307/1356563

Rosen, S.A. 1997, Lithics after the Stone Age. Walnut Creek, CA: Altamira Press, 184 p.

Rosen, S. 2013, Arrowheads, Axes, Ad Hoc, and Sickles: An Introduction to Aspects of Lithic Variability across the Near East in the Bronze and Iron Ages. Lithic Technology, 38(3): 141-149. doi:10.1179/0197726113Z.00000000023

Rosen, S., \& Vardi, J. 2014, Appendix IIH: The Chipped Stone Assemblage from Be'er Resisim-A Final Report. In: Excavations at the Early Bronze Age IV sites of Jebel Qa'Aqir and Be'er Resisim (Dever, W.G., Ed.), Eisenbrauns, Winona Lake, IN: p. $327-$ 338. URL: http://www.academia.edu/6491264/

Rowan, Y., \& Levy, T.E. 1991, Use Wear Analysis of Chalcolithic Scraper Assemblage from Shiqmin. Mitekufat Haeven: Journal of the Israel Prehistoric Society, 1991: 112-134.

Semenov, S.A. 1964, Prehistoric Technology. Barnes and Noble, New York, 211 p.

Sherratt, A.G. 1997a, Plough and Pastorialism: Aspects of the Secondary Products Revolution. In: Economy and Society in Prehistoric Europe: Changing Perspectives (Sherratt, A., Ed.), Princeton University Press, Princeton: p. 158-198.

Sherratt, A.G. 1997b, The Secondary Exploitation of Animals in the Old World. In: Economy and Society in Prehistoric Europe: Changing Perspectives (Sherratt, A., Ed.), Princeton University Press, Princeton: p.199-228.

Sliva, R.J., \& Keeley, L.H. 1994, "Frits" and Specialized Hide Preparation in the Belgian Early Neolithic. Journal of Archaeological Science, 21: 91-99. doi:10.1006/jasc.1994.1010

Stepien, U. 2012, Tabular Scrapers from the Eastern Kom at Tell el-Farkha. Studies in Ancient Art and Civilization, 16: 75-84. 
Unger-Hamilton, R. 1991, The Microwear Analysis of Scrapers and "Sickle Blades." In: Excavations at Jawa 1972-1986: Stratigraphy, Pottery, and Other Finds (Betts, A.V.G., Ed.), Excavations and Explorations in the Hashemite Kingdom of Jordan Vol. 1, Edinburgh University Press, Edinburgh: p. 149-153, 351.

Vila, E., \& Helmer, D. 2014, The Expansion of Sheep Herding and the Development of Wool Production in the Ancient Near East: An Archaeozoological and Iconographical Approach. In: Wool Economy in the Ancient Near East and the Aegean: From the Beginnings of Sheep Husbandry to Institutional Textile Industry (Breniquet, C., \& Michel, C. Eds.), Oxbow Books, Oxford: p. 22-40.

Wilkinson, T. C., Sherratt, S., \& Bennet, J., (Eds.), 2011, Interweaving Worlds: Systemic Interactions in Eurasia 7th-1st Millennia B.C., Oxbow Books, Oxford, 308 p.

Yamada, S. 2012, Use-wear analysis of tools from Nahal Zehora I. In: Village Communities of the Pottery Neolithic Period in the Menashe Hills, Israel: Archaeological Investigations at the sites of Nahal Zehora (Gopher, A., Ed.), Emery and Claire Yass Publications in Archaeology Vol. 29, Institute of Archaeology, Tel Aviv University, Tel Aviv: p. 9781010.

Yeivin, E., \& Olami, Y. 1979, Nizzanim - A Neolithic Site in Nahal Evtah: Excavations of 1968-1970. Tel Aviv, Journal of the Institute of Archaeology, 6(3-4): 9-135. doi:10.1179/033443579788441154

Yerkes, R.W. 1987, Prehistoric Life on the Mississippi Floodplain: Stone Tool Use, Settlement Organization, and Subsistence Practices at the Labras Lake Site, Illinois. University of Chicago Press, Chicago, 279 p.

URL: http://press.uchicago.edu/ucp/books/book/chicago/P/bo5965622.html

Yerkes, R.W., \& Barkai, R. 2012, Microwear analysis of bifacial tools at Nahal Zehora. In: Village Communities of the Pottery Neolithic Period in the Menashe Hills, Israel: Archaeological Investigations at the sites of Nahal Zehora (Gopher, A., Ed.), Emery and Claire Yass Publications in Archaeology 29, Institute of Archaeology, Tel Aviv University, Tel Aviv: p. 930-977.

Yerkes, R.W., \& Kardulias, P.N., 1993. Recent Developments in the Analysis of Lithic Artifacts. Journal of Archaeological Research, 1: 89-119. doi:10.1007/BF01326933

Zutovski, K., Yerkes, R.W., Agam, A., Willson, L., Getzov, N., Milevski, I., \& Gopher, A. 2016, A techno-typological analysis of fan (tabular) scrapers from Ein Zippori, Israel. Journal of Lithic Studies, 3(1): 32 p. doi:10.2218/jls.v3i1.1454 